Cahiers $d u$ MONDE RUSSE

\section{Cahiers du monde russe}

Russie - Empire russe - Union soviétique et États indépendants

$58 / 4 \mid 2017$

Varia

\title{
Molly Brunson, Russian Realisms, Literature and Painting, 1840-1890
}

\section{Cécile Pichon-Bonin}

\section{OpenEdition}

\section{Journals}

Édition électronique

URL : http://journals.openedition.org/monderusse/10195

DOI : 10.4000/monderusse.10195

ISSN : $1777-5388$

\section{Éditeur}

Éditions de l'EHESS

\section{Édition imprimée}

Date de publication : 1 octobre 2017

Pagination : 751-754

ISBN : 978-2-7132-2698-4

ISSN : $1252-6576$

Référence électronique

Cécile Pichon-Bonin, « Molly Brunson, Russian Realisms, Literature and Painting, 1840-1890 》, Cahiers du monde russe [En ligne], 58/4 | 2017, mis en ligne le 01 octobre 2017, consulté le 06 janvier 2021.

URL : http://journals.openedition.org/monderusse/10195; DOI : https://doi.org/10.4000/monderusse. 10195

(c) École des hautes études en sciences sociales 


\section{Molly BRUNSON}

\section{Russian Realisms, Literature and Painting, 1840-1890}

DeKalb : Northern Illinois University Press, 2016, 263 p.

Dans cet ouvrage, Molly Brunson étudie les relations interartielles entre la peinture et la littérature russes des années 1840-1890. Dépassant une simple confrontation ou comparaison entre ces deux médias, l'auteure n'entend pas donner une définition absolue du visuel et du verbal, mais bien saisir comment «l'autre » artistique est imaginé dans une œuvre et comment artistes et auteurs interrogent ainsi les capacités de représentation de ces médias. Elle fait un triple pari, réussi : par cette approche, mieux comprendre le réalisme, réhabiliter la peinture réaliste russe longtemps méprisée par la critique d'art occidentale, notamment moderniste, et lui donner ainsi sa place parmi les réalismes européen et américain.

L'auteure conçoit le réalisme comme un phénomène à la fois transhistorique et historique et établit un dialogue fécond entre esthétique, histoire de l'art et histoire de la littérature. Plaçant l'analyse des œuvres au centre de son propos, elle postule que l'étude des relations interartielles, inhérentes au réalisme transhistorique, constitue la clé conceptuelle permettant de définir les conventions esthétiques du réalisme russe du XIX ${ }^{e}$ siècle ainsi que l'idéologie et les buts métaphysiques des écrivains et peintres de cette époque (p. 3-4). 
En outre, elle envisage le réalisme non comme une fin en soi, mais bien comme un projet, ou une aspiration, traversé de débats (comme celui sur la forme et le contenu), de tensions et de contradictions que les œuvres révèlent. Molly Brunson attire notamment l'attention sur le caractère conscient du projet réaliste dans son ensemble. Tout en étant lucides sur l'impossibilité de parvenir à l'équivalence mimétique parfaite, les artistes poursuivent leurs recherches et investiguent en profondeur les rapports de l'art à la réalité. Alors que les Réalistes du XIX ${ }^{\mathrm{e}}$ siècle persistent volontairement dans ce projet de représentation, les artistes modernes renoncent et ouvrent d'autres voies. Ce serait là l'une des grandes différences entre ces tendances. L'ouvrage prend le contre-pied des critiques modernistes alléguant que le réalisme se perd dans une recherche imitative ignorant le médium et les conventions artistiques et démontre au contraire que les artistes réalistes affirment le caractère autoréférentiel de l'œuvre, notamment peinte, et que cette démarche s'intègre bien dans une réflexion sur la représentation artistique. De l'analyse, émergent les procédés mis au point par les auteurs et les peintres pour, à la fois, convoquer la réalité et interroger le processus de représentation de cette réalité.

Le livre se découpe en cinq chapitres proposant chacun des études de cas détaillées et convaincantes, suivant une progression chronologique. Le premier analyse l'interrelation entre le visuel et le verbal, à travers le thème de la fenêtre dans quelques œuvres de l'école naturaliste : des textes tirés de La Physiologie de Pétersbourg (1845), rédigés notamment par Dmitrij Grigorovič ou Vissarion Belinskij, et la peinture de genre de Pavel Fedotov, en particulier son tableau Les Fiançailles $d u$ major (ou La Demande en mariage du major endetté) (1848). Le deuxième chapitre étudie l'expression du motif de la route dans des œuvres jugées représentatives de la période de la réforme des années 1850-1860, à savoir la première prose d'Ivan Turgenev et la peinture de genre de Vasilij Perov. L'auteure consacre son troisième chapitre à l'illusion romanesque chez Tolstoj en étudiant principalement la bataille de Borodino dans Guerre et Paix (1865-1869) alors que, dans le quatrième, elle analyse plusieurs tableaux d'Il'ja Repin: Les Haleurs de la Volga (1870-1873), Ivan le Terrible tue son fils (1883-1885) et Les cosaques zaporogues écrivant une lettre au sultan de Turquie (1891). Enfin, le cinquième chapitre porte sur l'image réaliste chez Fëdor Dostoevskij et se focalise essentiellement sur L'Idiot (1869).

Tout au long du livre, Molly Brunson définit ainsi les types de relations qu'entretiennent le verbal et le visuel dans les œuvres littéraires et picturales, ce qu'elle nomme le paragone réaliste, et leurs différentes fonctions. Pour ce faire, elle analyse les sujets et les procédés (technique, composition, espace, touche, couleur pour le visuel, ou narration, intrigue, enargeia, description et ekphrasis pour le verbal). Elle distingue ainsi la coopération, jusqu'à l'inclusion, du verbal et du visuel dans la mimesis multimodale de l'école naturaliste, la différenciation opérée par Perov et Turgenev, la querelle s'exprimant chez Tolstoj, qui proclame la supériorité du roman sur la peinture et, dans une veine moins polémique, chez Repin, et enfin la fusion réalisée par Dostoevskij, dans un but de transfiguration de ce verbal et de ce visuel.

L'analyse se fonde sur un corpus de sources primaires riches et variées, parmi lesquelles on compte tableaux, illustrations, travaux préparatoires, esquisses, 
mémoires, correspondances, manuscrits, notes des auteurs, comptes rendus critiques. L'auteur interroge aussi bien les œuvres achevées que les processus de création des écrivains et artistes, le choix de leurs modèles, leur façon d'observer, d'enquêter, de reconstituer les lieux et les événements. À cela s'ajoutent des sources secondaires principalement théoriques et philosophiques (qui vont d'Horace et Lessing à Ernst Gombrich, Roland Barthes ou surtout William J.T. Mitchell). L'ouvrage est émaillé de points historiographiques qui permettent à l'auteur de situer sa pensée dans le champ des études d'histoire de l'art sur le réalisme russe.

Si le caractère multiforme du réalisme a été observé depuis longtemps, le fait de l'analyser sous l'angle des relations interartielles permet de fonder cette diversité. Cette approche rend évidents les nuances, différences et points communs entre les projets réalistes qui se développent à chaque période.

Et cet angle de vue présente bien d'autres mérites. La méthode permet aussi de revisiter des grandes problématiques réalistes et de montrer précisément comment les réflexions formelles et chaque projet esthétique s'articulent avec un projet éthique, professionnel, une promotion de la culture nationale, un engagement social, une idéologie politique et/ou l'espoir d'une transfiguration spirituelle. L'auteure relie ainsi la stratégie interartielle collaborative de l'école naturaliste et de Fedotov à leur idéal d'égalité démocratique et la différenciation de Turgenev et Perov à l'éclatement des sphères sociale et politique à l'époque de la réforme. Chez Dostoevskij, il s'agit plutôt de montrer la corrélation entre objectifs esthétiques et religieux. Loin d'être simpliste, l'analyse propose différents niveaux de lecture pour chaque œuvre. Les relations interartielles dans les tableaux de Repin sont ainsi étudiées comme expression de positions sur des questions sociales et sur la place de l'artiste par rapport à ces sujets, ou encore comme affirmation de la dimension matérielle et autoréférentielle de la peinture et d'une culture nationale russe. Les œuvres sont comprises dans leur contexte historique et dans une perspective transhistorique plus large. L'usage du visuel dans les romans de Tolstoj et Dostoevskij est ainsi éclairé par le développement de l'optique au $\mathrm{XIX}^{\mathrm{e}}$ siècle, en particulier les lanternes magiques et la photographie, tout autant que par des références à des figures mythologiques comme Méduse ou la Rusalka.

De nombreux procédés sont ainsi mis au jour et/ou expliqués de façon nouvelle. Molly Brunson éclaire les motivations qui ont présidé au choix et au traitement des sujets historiques et mythologiques par les réalistes russes, spécificité qui a longtemps laissé perplexes les commentateurs, notamment occidentaux. Elle met en évidence l'importance et la fonction de la profondeur émotionnelle et psychologique dans les œuvres réalistes russes; elle analyse finement la place et le rôle dévolus au spectateur ou au lecteur, que celui-ci se trouve devant la fenêtre de l'école naturaliste ou face à la croisée des chemins du réalisme critique des années 1860 .

L'étude des relations interartielles permet en effet de préciser les pratiques, fonctions, ambitions, limites et outils de la littérature et de la peinture dans leurs spécificités et dans leur rapport aux grandes notions qui hantent le réalisme, et cela constitue un autre atout de cet ouvrage. Plutôt que d'aborder ces notions de front, le regard est décentré de façon salutaire et l'auteure nourrit ainsi les réflexions sur les 
relations entre le réalisme et les concepts de vérité, d'illusion, d'espace et de temps. De la sorte, la question du rapport de l'art à la réalité, de l'art à la vie, fondement du réalisme, traverse tout l'ouvrage, essentiellement à travers l'exploration des capacités de représentation du visuel et du verbal. À la lecture de ce très riche opus, on se demande enfin si l'étude des relations interartielles pourrait achopper sur l'un des grands problèmes philosophiques inhérents au réalisme : la définition du réel, ou permettre de l'éclairer. Les écrivains et artistes convoqués ici partagent-ils vraiment la même définition? Le réalisme fantastique de Dostoevskij permet d'en douter. La position de l'auteur sur ce sujet présenterait à nos yeux un réel intérêt.

Cécile Pichon-Bonin

CNRS, centre Georges Chevrier 